\title{
Pre-service Middle School Mathematics Teachers' (Mis)conceptions of Definitions, Classifications, and Inclusion Relations of Quadrilaterals
}

\author{
Gamze Baktemur ${ }^{1}$, Rukiye Ayan Civak ${ }^{2 *}$, Mine Isiksal Bostan ${ }^{3}$ \\ ${ }^{1}$ Ministry of National Education, TURKEY \\ 2 Izmir Democracy University, TURKEY \\ ${ }^{3}$ Middle East Technical University, TURKEY \\ * Corresponding author: rukiye.ayancivak@idu.edu.tr
}

Received: 25 May 2021 Accepted: 1 Jul. 2021

Citation: Baktemur, G., Ayan Civak, R., \& Isiksal Bostan, M. (2021). Pre-service Middle School Mathematics Teachers' (Mis)conceptions of Definitions, Classifications, and Inclusion Relations of Quadrilaterals. European Journal of Science and Mathematics Education, 9(4), 183-198. https://doi.org/10.30935/scimath/11206

\begin{abstract}
:
The purpose of this study is to examine pre-service middle school mathematics teachers' (mis)conceptions related to definitions, classifications, and inclusion relations of convex quadrilaterals through a case study research design. The participants of the study were 20 pre-service middle school mathematics teachers who attended a must course, "The Methods of Teaching Mathematics" in a public university in Ankara, Turkey. Purposive sampling strategy was used to select the participants. The data were collected through an achievement test before and after taking the methods of teaching mathematics course. The findings of the study revealed that pre-service teachers had misconceptions in hierarchical classification of quadrilaterals, dual inclusion relations (e.g., rhombus-trapezoid), and difficulty in formulating minimal definitions of quadrilaterals before taking the course. However, the findings also indicated that the course had a positive impact for eliminating those misconceptions and supporting their conceptions. Several suggestions were made based on these findings.

Keywords: pre-service middle school mathematics teachers, definitions of quadrilaterals, classifications of quadrilaterals, inclusion relations of quadrilaterals
\end{abstract}

\section{INTRODUCTION}

Content knowledge (Shulman, 1987) or common content knowledge (Ball, Thames, and Phelps, 2008) is an important type of knowledge for teachers since they should know particular topics that they will teach. According to Ball et al. (2008), teachers should know the rules, facts, and concepts in these topics as well as the underlying reasons of them. National Council of Teachers of Mathematics [NCTM] (2000) identified five content standards for mathematics education that are numbers and operations, algebra, geometry, measurement, and data analysis and probability. Each standard has specific aims and importance in school mathematics. In particular, geometry is considered as an important content standard since it enhances students' spatial reasoning, which is related to understanding spatial relations, spatial orientations and visualization (Clements \& Battista, 1992; Jones \& Tzekaki, 2016). In this respect, mathematics teachers should have a conceptual knowledge of shapes and their properties, transformation, location and visualization, which constitute the general content goals of geometry (Van de Walle, Karp, \& Bay-Williams, 2013). Within the context of shapes and properties, special quadrilaterals (the term quadrilaterals will be used to refer to special quadrilaterals throughout the rest of the paper to avoid repetition), including (kite, trapezoid, parallelogram, rectangle, rhombus, and 
square) constitute one of the important topics. Especially the inclusion relations between and among these quadrilaterals (e.g., every parallelogram is a trapezoid) are essential for conceptual understanding and geometrical thinking (Fujita \& Jones, 2007).

In Turkey, the topic of quadrilaterals is taught in the seventh grade throughout 15 hours, and the curriculum has a sub-objective for inclusion relations of quadrilaterals (Ministry of National Education [MoNE], 2018). However, a pile of studies has shown that students, pre-service and in-service teachers have various difficulties related to the classifications, definitions, and inclusion relations of quadrilaterals (Akkaş \& Türnüklü, 2015; Aktaş \& Cansız-Aktaş, 2012; Fujita \& Jones, 2006; Karakuş \& Erşen, 2016; Loc, Tog, \& Hai, 2017; Özkan \& Bal, 2017; Türnüklü, 2014; Zeybek, 2017; Zilkova, 2015). For instance, $95 \%$ of the primary school students claimed that a square is not a rectangle (Loc et al., 2017). Furthermore, Türnüklü (2014) reported that only one pre-service middle school mathematics teacher (PMSMT) out of 15 established a correct relationship between rhombus and parallelogram (e.g., every rhombus is a parallelogram). Therefore, the research addresses the gaps in teachers' knowledge in classifications, definitions, and inclusion relations of quadrilaterals and calls for the need for improving teachers' content knowledge.

Many studies showed that methods courses contribute to the understanding of pre-service teachers' content and pedagogical content knowledge in number and operations, geometry, measurement, algebra, probability, and statistics (Matthews, Rech, \& Grandgenett, 2010; Quinn, 1997; Smith et al., 2012; Youngs \& Qian, 2013). To illustrate, Quinn (1997) studies with 47 PMSMTs and showed that their content knowledge in place value, percentage, fractions, multiplication, division, estimation, area, perimeter, geometry, number lines, measurement, probability, and statistics improved substantially at the end of the methods course. As aforementioned, studying of classifications and inclusion relations of quadrilaterals is significant. However, even though it is reported that methods course enhances preservice teachers' content knowledge in most content areas research that solely focuses on examining whether methods course helps pre-service teachers improve their content knowledge in inclusion relations of quadrilaterals is scarce. Therefore, the purpose of this study is to investigate PMSMTs' conceptions and (mis)conceptions- related to definitions, classifications, and inclusion relations of quadrilaterals before and after taking the methods of teaching mathematics course.

\section{Theoretical and Conceptual Background}

Shulman (1987) identified seven categories of knowledge that teachers should have. One of these categories is Content Knowledge (CK) that puts an emphasis on teachers' knowledge of the subjects they will teach. Pedagogical Content Knowledge (PCK) is content knowledge that is required for teaching (Shulman, 1986). PCK includes teachers' conceptions related to how students learn topics (Park \& Oliver, 2008). Like Shulman, Ball et al. (2008) attached importance to teacher knowledge and identified four domains for teacher knowledge: Common Content Knowledge (CCK), Specialized Content Knowledge (SCK), Knowledge of Content and Students (KCS), and Knowledge of Content and Teaching (KCT). CCK is not specific to teaching but related to the mathematical knowledge necessary to teach (Ball et al., 2008). KCS is a combination of knowing students (e.g., their (mis)conceptions) and mathematical topics (Ball et al., 2008). In teaching the mathematics content standards, CCK and KCS are especially important in order to create an effective learning environment.

When the geometry standard in school mathematics and teacher knowledge are considered together, teachers should master at geometrical topics such as shapes and their properties, know how to introduce each topic to students conceptually, and anticipate possible misconceptions that may come from their students. Therefore, specific to the classification of quadrilaterals and inclusion relations among them, teachers should know the definitions and properties of each quadrilateral, how quadrilaterals relate to each other in terms of inclusion relations, how to teach these conceptually, and to be able to anticipate possible misconceptions of their students related to the classification and inclusion relations. 


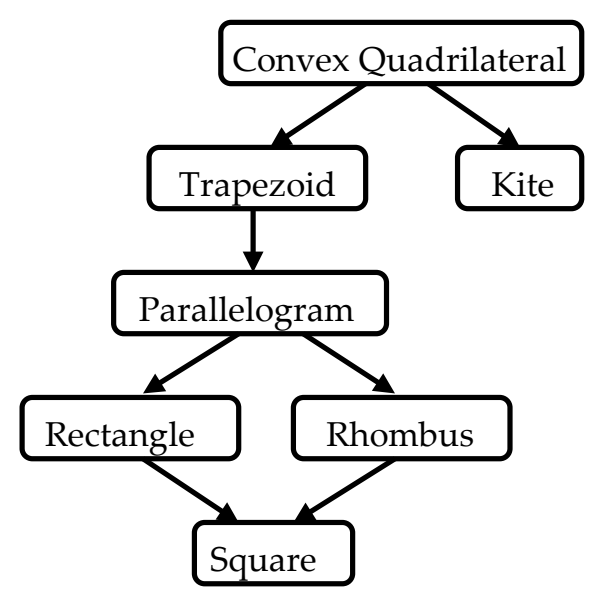

Figure 1. Hierarchical classification of convex quadrilaterals

There are four types of classifications for quadrilaterals: Partition, Hierarchical, Descriptive, and Constructive (De Villiers, 1994). In the Partition Classification, there is no emphasis on the relations among the shapes whereas shapes are classified in such a way that particular concepts are considered as subsets of more general concepts in the Hierarchical Classification. Moreover, in the Descriptive (posteriori) Classification an emphasis on the classification of the shapes comes after making explorations with shapes and their properties for a substantial amount of time. Lastly, an inductive approach that includes a consecutive progress from the most special shape (i.e., square) to more general shapes (i.e., parallelogram, trapezoid) is followed in the Constructive (priori) Classification. In this study, the Hierarchical Classification of quadrilaterals was adopted to make sense of the participants' (mis)conceptions since it puts an emphasis on the inclusion relations among quadrilaterals, which is illustrated in Figure 1.

Even though no study was encountered in the available literature that focuses on pre-service teachers' knowledge regarding the inclusion relations among quadrilaterals before and after taking a methods course, a number of studies were conducted to examine teachers' knowledge and misconceptions in quadrilaterals and their inclusion relations through a one-shot data collection process. To begin with, it is reported in several studies that pre-service teachers had misconceptions in dual relations of quadrilaterals (Fujita \& Jones, 2006; Karakuş \& Erşen, 2016; Türnüklü, 2014; Zilkova, 2015). For instance, only one out of 15 PMSMTs was able to make an association between a parallelogram and a rhombus, and none of the participants mentioned the inclusion relations between a rectangle and a rhombus (Türnüklü, 2014). In addition, pre-service primary education teachers had problems related to inclusion relations between a kite and a quadrilateral, a rectangle and a parallelogram, and a square and a rhombus (Zilkova, 2015). Other studies reported that only $8.9 \%$ of the trainee primary teachers were able to identify a square as a trapezoid (Fujita \& Jones, 2006) and $63.16 \%$ of the pre-service primary school teachers accepted the statement "all rectangles are trapezoid" as incorrect (Karakuş \& Erşen, 2016). Moreover, a few studies showed that pre-service teachers had misconceptions in definitions of and hierarchical classifications among quadrilaterals (Erdoğan \& Dur, 2014; Karakuş \& Erşen, 2016). In relation to this, it was showed that pre-service mathematics teachers could not define quadrilaterals hierarchically (Erdoğan \& Dur, 2014) and $62.07 \%$ of the pre-service primary school teachers were not able to define a trapezoid (Karakuş \& Erşen, 2016).

In addition to the studies that assess pre-service teachers' knowledge and misconceptions in properties of quadrilaterals and their inclusion relations through a one-shot data collection process, a few studies also investigated pre-service teachers' progress in these subjects throughout a time period (Ulusoy, 2016; Zeybek, 2017). For example, Zeybek (2017) revealed that at the beginning of the semester $60 \%$ of the pre-service mathematics teachers showed partial prototypical reasoning, and they had many problems in accepting other quadrilaterals as trapezoids. On the other hand, the findings of the study showed a significant increase in participants' hierarchical reasoning at the end of the semester in such 
a way that $62 \%$ of them were able to classify quadrilaterals hierarchically. In another intervention study, Ulusoy (2016) found that a rectangle was not thought as a specific case of a parallelogram by pre-service mathematics teachers at the beginning of the intervention. However, the findings showed that the preservice teachers were able to enhance their subject matter knowledge throughout the intervention.

To sum up, the research (Akkaş \& Türnüklü, 2015; Erdoğan \& Dur, 2014; Fujita \& Jones, 2006; Öztoprakçı, 2014; Zilkova, 2015) that focuses on pre-service and in-service teachers' knowledge of and misconceptions in definitions, classifications and inclusion relations of quadrilaterals has consistently showed that preservice mathematics teachers $\mathrm{s}$ and primary education teachers have poor conceptions and several misconceptions in definitions, classifications, and inclusion relations of quadrilaterals. On the other hand, the findings of a few studies also showed that PMSMTs' knowledge could be improved, and their misconceptions could be overcome through specific training and interventions (Ulusoy, 2016; Zeybek, 2017). Improving teachers' knowledge and eliminating their misconceptions related to definitions, classifications, and inclusion relation of quadrilaterals is significant in the sense that if these misconceptions are eliminated and more comprehensive and reach conceptions are established, preservice teachers could notice and overcome students' existing misconceptions and design their instruction based on those misconceptions. To this end, we examined PMSMTs' conceptions and misconceptions related to definitions, classifications, and inclusion relations of quadrilaterals before and after taking the methods of teaching mathematics course. More specifically, in this research, we identified PMSMTs' development in these respects by examining their responses to the achievement test prepared by the researchers before and after taking the methods of teaching mathematics course.

\section{METHODS AND PROCEDURES}

\section{Design of the Study}

The design of the study was case study, whose typical characteristics include focusing on a case that can be a single individual or a group of individuals like a class, event or process and studying with this case (Fraenkel, Wallen, \& Hyun, 2012; Yin, 2003). In this study, there was a specific case of PMSMTs attending to the methods of teaching mathematics course, and the study focused on their (mis)conceptions related to definitions, classifications, and inclusion relations of quadrilaterals before and after taking this course.

\section{Participants}

The study was conducted with 20 pre-service teachers who were junior students at the elementary mathematics education program in a public university in Ankara, in the Spring semester of 2018-2019 academic year. The medium of instruction in this university is English. This university is one of the highest-ranking universities in Turkey. Students need to get high scores in the national university entrance exam and be among the top students to study in this university. The sample of the study was selected by using a typical sample, which is a type of purposive sampling. A typical sample is a group of participants whose characteristics are representative for the study (Fraenkel, Wallen, \& Hyun, 2012). There were 18 females and 2 males whose ages ranged from 20 to 22 in the study sample. In the previous years, these students had taken pure mathematics courses (Fundamentals of Mathematics, Analytic Geometry, Calculus, Discrete Mathematics, (Linear) Algebra, Introduction to Differential Equations, Elementary Geometry) and Introduction to Probability and Statistics prior to the study. They had also taken a few educational courses (Introduction to Education, Educational Psychology, etc.). However, they were taking their second mathematics education course (Methods of Teaching Mathematics I and II) at the time of the study. The details of these methods courses are provided in the following section.

\section{The Course and the Context}

The elementary mathematics education program where the data were collected has two Methods of Teaching Mathematics Courses in the third year of the program in two semesters. Within the context of the Methods of Teaching Mathematics Course II conducted in the second semester of the 2018-2019 
academic year, the participants of this study attended to weekly lessons (four hours every week) in two sessions conducted on two days through 13 weeks. In these lessons, they learned how each content standard (number and operations, algebra, geometry, measurement, and data analysis and probability) can be taught to middle school students conceptually. In particular, two weeks (eight class hours) were devoted to teaching the domain of geometry, which is the focus of this study. In this two-week-period, the instruction focused on the van Hiele Levels of Geometric Thought, two dimensional and threedimensional shapes and their properties, activities related to shapes based on van Hiele Levels, transformations, and visualization. More specifically, an inquiry-based mathematics education approach (Artigue \& Blomhøj, 2013) was followed in which PMSMTs were engaged in discussions related to essential elements of these subjects, and they practiced how to design and implement tasks, problems, and activities to support conceptual understanding of students in classrooms (Jaworski, 2004). In these courses, the main textbook was Elementary and middle school mathematics: Teaching developmentally (van de Walle et al., 2013)

\section{Data Collection Instruments}

The data for this study were collected by the Convex Quadrilateral Test, which was developed by the researchers based on the related studies in the literature (e.g., Brunheira, \& da Ponte, 2019; De Villiers, 1994) and the content of the main textbook of the course (van de Walle et al., 2013). This test included various types of questions including open-ended and true-false questions related to definitions of quadrilaterals, inclusion relations among these quadrilaterals (including dual relations), identifying the given shapes as special quadrilaterals, and drawing alternative quadrilaterals (other than prototypes). These questions were prepared in order to explore how PMSMTs classified quadrilaterals, established relationships among them and whether or not they defined quadrilaterals by using inclusion relations. Expert opinion was taken from a professor in mathematics education for content-related evidence of validity to ensure compatibility of content of the questions. Besides, for the purposes of validity, a table of specification through which the consistency between the questions on the test and the objectives of the Turkish Middle School Mathematics Curriculum was constructed in order to see which questions measured which objectives. The same test was implemented twice (as pre-test and post-test) before and after the participants took the two-week-period of Methods of Teaching Mathematics Course, which focused on teaching geometry. This test was given as an Appendix to this study.

\section{Data Collection Procedures}

The data were collected from a class of PMSMTs who were taking Methods of Teaching Mathematics II course in the 2018-2019 Spring semester at two occasions, two weeks apart - before and after being instructed on geometry and how to teach geometry within the context of the course. The pre-test was implemented as a paper-pencil test while the post test was implemented online. The reason for this divergence was due to the convenience for the researchers and the participants, and the fulfilment of the requirements of the course. Necessary permissions were taken from the relevant institution and the participants, and the participants were fully informed about the purposes and the procedures of the study beforehand. They were also informed that the participation was fully based on voluntariness. The data collected from the PMSMTs were only analyzed in line with the purposes of the study, and full confidentiality was assured both in the processes of data collection and analysis and reporting the findings of the study.

\section{Data Analysis}

The data were analyzed under the three sub-themes: classification of quadrilaterals, dual inclusion relations of quadrilaterals, and definitions of quadrilaterals in relation to the research questions of the study. Content analysis, which includes analyzing people's behaviors by using their communications (Fraenkel et al., 2012), was used for the analysis of the data collected. Particularly, each participant's answers to the pre-test were investigated in terms of their (correct) conceptions and misconceptions. 
More specifically, in line with the research questions of the study, participants' concept maps for the first question were explored in terms of their accuracy related to the inclusion relations among the quadrilaterals (see Figure 1). Besides, their concept maps were explored in order to see incorrect inclusion relations among the quadrilaterals. Each time a new incorrect inclusion relation was observed in the concept maps drawn by the participants, it was recorded until there was no new incorrect relation, which showed that the data were saturated. Participants' responses to the second question was investigated in terms of whether or not they were able to identify the true statements related to the inclusion relations as true or false statements as false, which provided us with a frequency of accurate and inaccurate answers to each sub-question to see the patterns in participants' incorrect answers. Moreover, the revised statements provided by them for the questions they identified as false were also investigated in terms of inclusion relations among the quadrilaterals. The patterns in these revised statements were also recorded. Lastly, participants' responses to the third and the last question were investigated in terms of their accuracy; that is, whether or not they were able to correctly identify the given shapes. In addition, their alternative drawings were also investigated in order to see the patterns in them. These processes were followed for the analysis of PMSMTs' responses in both the pre-test and post-test. As the last phase of the data analysis process, participants' answers from the pre-test and posttest implementations were compared/contrasted in order to see the changes in their (mis)conceptions from the pre-test to the post-test. For the dependability of the study, the data were examined both by researcher and a co-coder who was a middle school mathematics teacher who did not take part in this study.

\section{FINDINGS}

The findings are presented in three sub-themes including classifications of all quadrilaterals, dual inclusion relations of quadrilaterals, and definitions of quadrilaterals in the following sections. Particularly, participants' development in those will be explained by a cross comparison of their performances in the pre- and post-test implementations.

\section{Classifications of Quadrilaterals}

When PMSMTs' work related to drawing a concept map in order to show the relationships among convex quadrilaterals on the pre-test was examined, it was seen that PMSMTs had various problems in hierarchical classification of quadrilaterals. Only two of the 20 PMSMTs were able to draw a concept map that correctly illustrated hierarchical inclusion relations among the quadrilaterals. Most of the incorrect inclusion relationships were related to the ones between a trapezoid and other quadrilaterals. On the other hand, when their work on the post-test was examined, it was seen that eight of the PMSMTs were able to correctly classify the quadrilaterals, except kite, hierarchically, and seven of them were able to draw correct concept maps including hierarchical relations among all the quadrilaterals. That is, a comparison of their performances on the pre-test and post-test revealed that PMSMTs enhanced their knowledge in classifying quadrilaterals throughout the methods course, and their misconceptions related to the hierarchical classification of quadrilaterals decreased.

A sample concept map drawn by PMSMT 10 on the pre-test is provided in Figure 2, in which she classified quadrilaterals in three categories as trapezoid, parallelogram, and kite. 


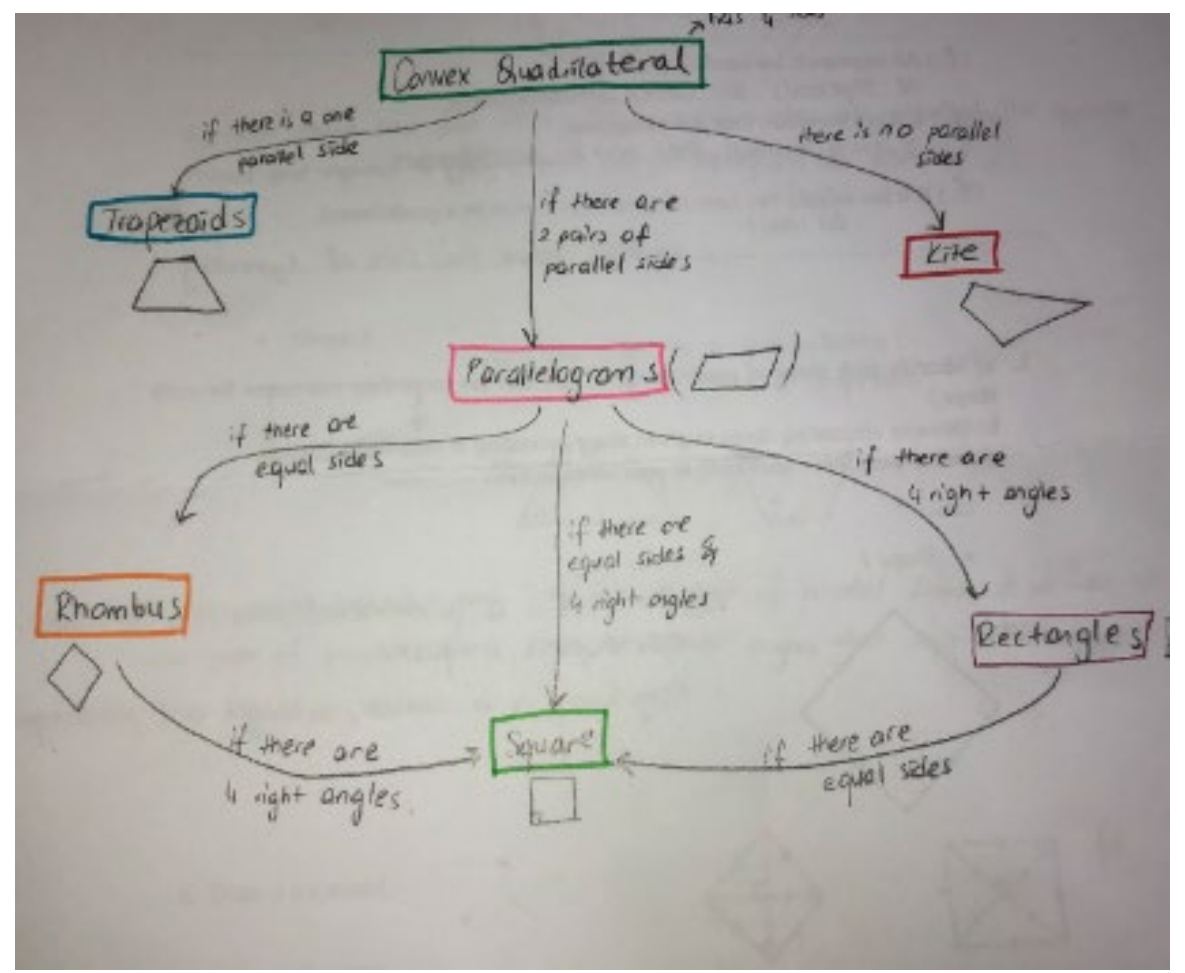

Figure 2. PMSMT 10's concept map on the pre-test

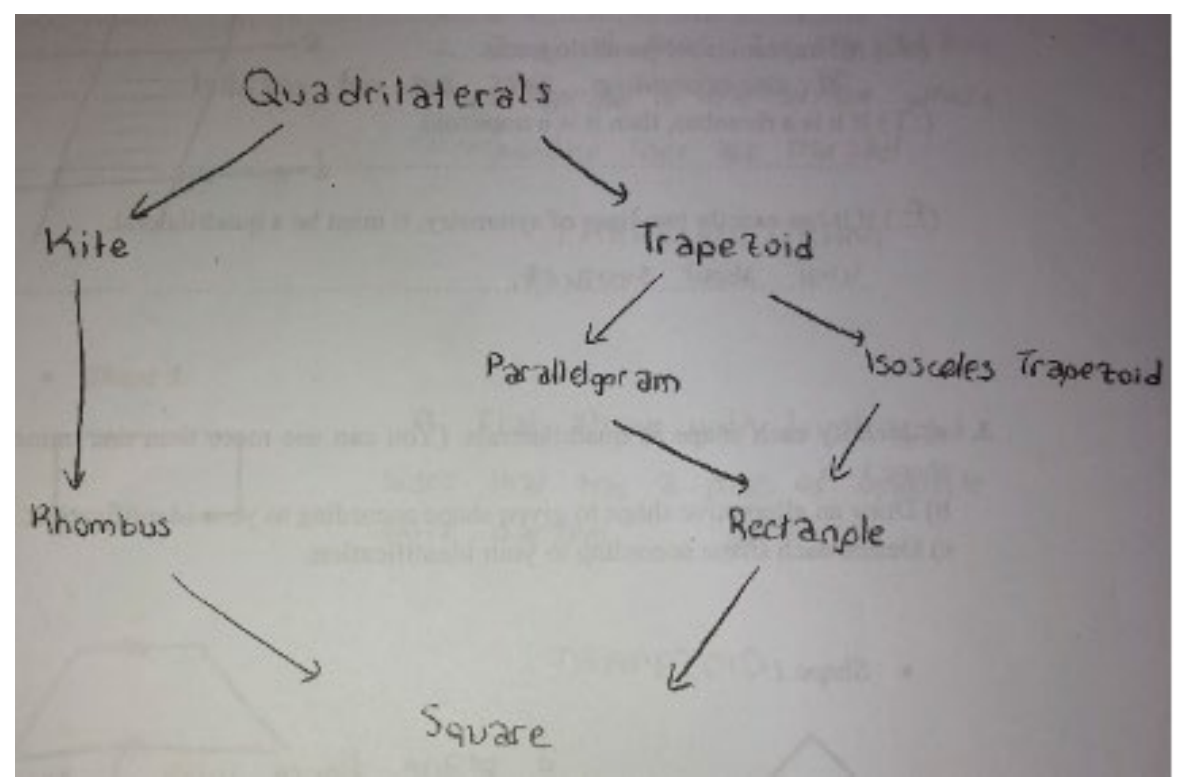

Figure 3. PMSMT 18's concept map on the pre-test

As understood from Figure 2, PMSMT 10 made a hierarchical classification including hierarchical relations among parallelogram, rectangle, rhombus, and square correctly. However, she did not establish any relationship between a parallelogram and a trapezoid. Another example of a concept map that included incorrect relationships drawn by PMSMT 18 is provided in Figure 3.

As understood from Figure 3, PMSMT 18 classified quadrilaterals in two categories as kite and trapezoid. Although she placed parallelogram, rectangle, and square correctly in the hierarchical concept map, she made an incorrect classification by placing rhombus under kite. In addition, although she correctly placed square as a special type of a rhombus, at the same time, she incorrectly placed square as a special type of kite. 


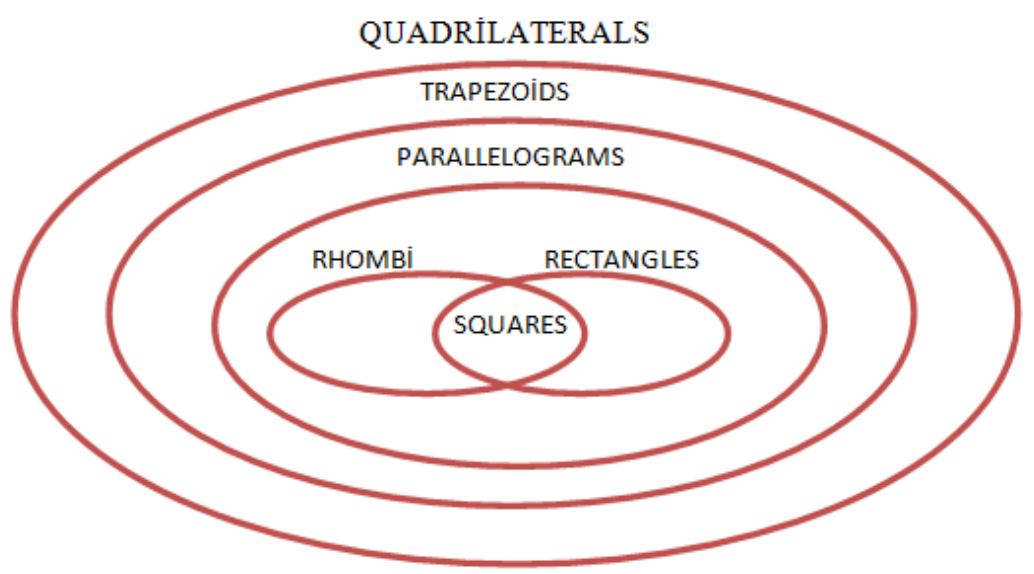

Figure 4. PMSMT 18's concept map on the post-test

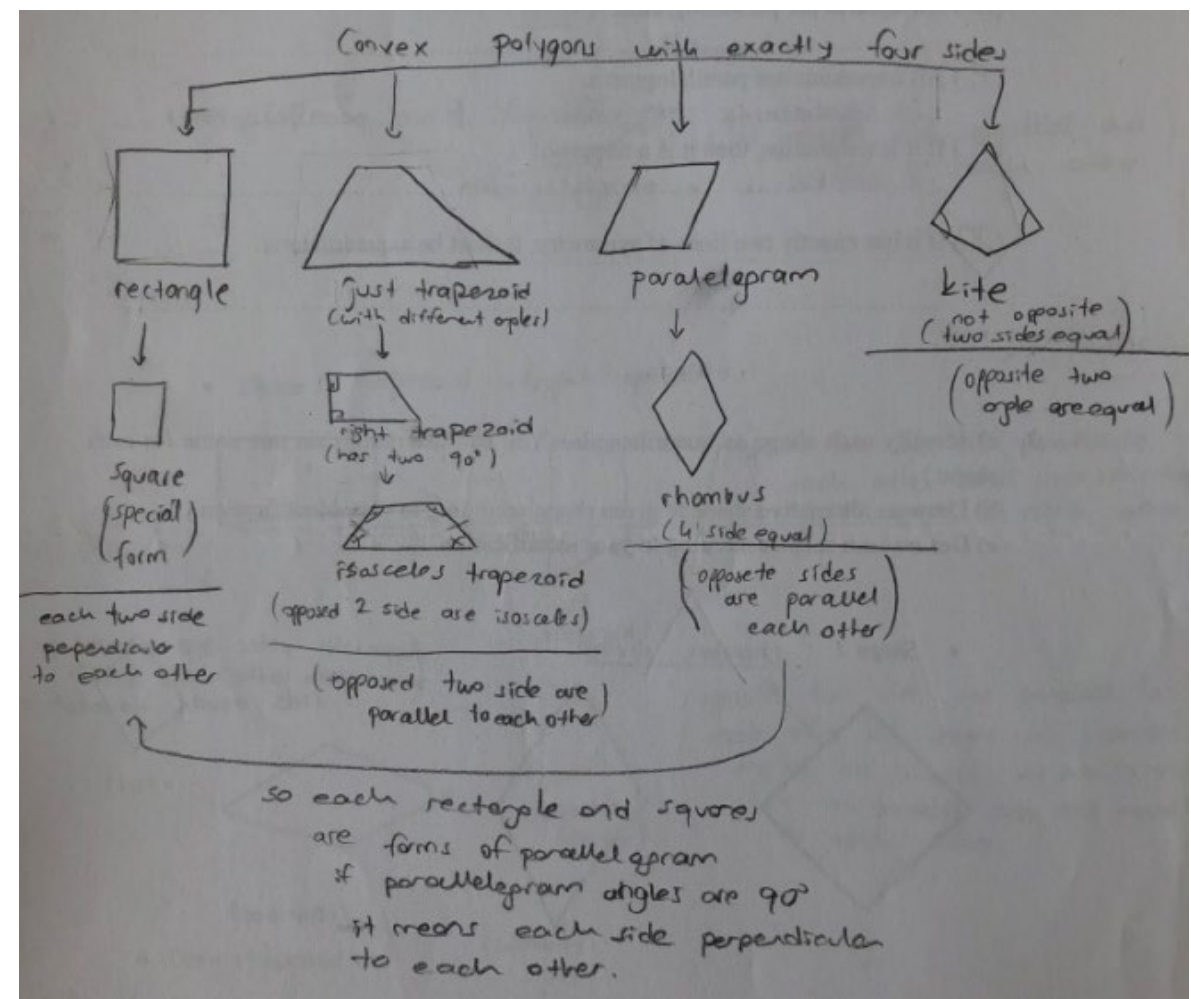

Figure 5. PMSMT 20's concept map on the pre-test

Both PMSMT 10 and PMSMT 18 corrected their misconceptions and drew correct concept maps on the post-test. For example, PMSMT 18's concept map included correct classification except kite on the posttest, and this is provided in Figure 4.

As understood from Figure 4, PMSMT 18 was able to place trapezoid as the most general form of quadrilaterals, parallelogram as a special form of a trapezoid, rhombus and rectangle as special forms of a parallelogram, and square as a special form of a rectangle and a rhombus. However, she did not include kite in any place in her concept map. In other words, PMSMT 18 was able to classify all quadrilaterals except kite correctly.

As another example of a concept map that included an incorrect hierarchical classification, the concept map drawn by PMSMT 20 could be given. In this map, PMSMT 20 put quadrilaterals into four categories as rectangle, trapezoid, parallelogram, and kite. The related concept map drawn by PMSMT 20 is provided in Figure 5. 


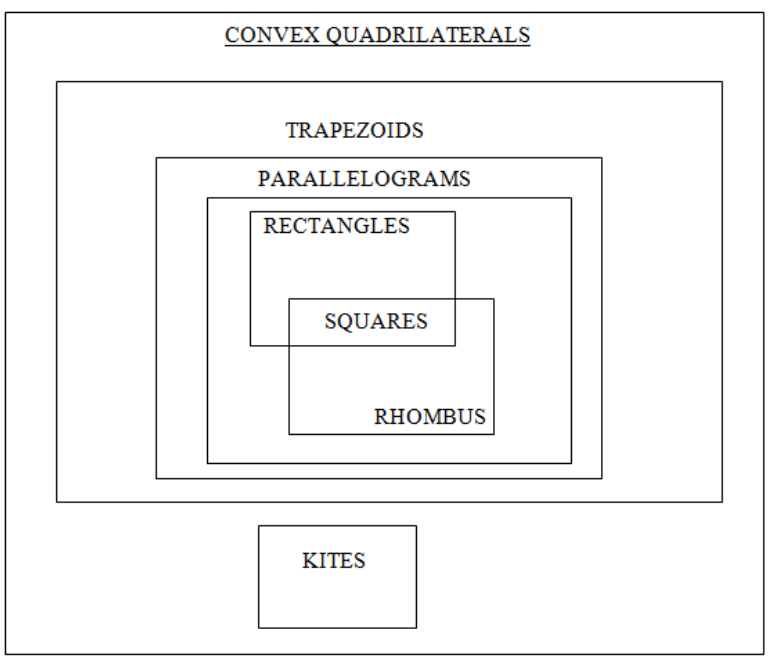

Figure 6. PMSMT 20's concept map on the post-test

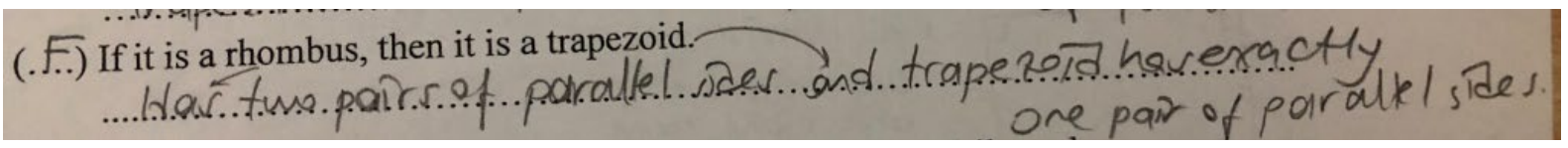

Figure 7. PMSMT 5's answer related to the rhombus-trapezoid relationship on the pre-test

As understood from Figure 5, PMSMT 20 correctly placed square as a special form of a rectangle and rhombus as a special form of a parallelogram. Also, she wrote that all rectangles and squares are special forms of a parallelogram, which is correct. However, she did not make any relationship between a trapezoid and other quadrilaterals. However, PMSMT 20's concept map included correct classifications on the post-test, is also provided in Figure 6.

As understood from Figure 6, PMSMT 20 divided quadrilaterals into two categories as trapezoid and kite. He was able to place parallelogram as a special form of a trapezoid, rhombus and rectangle as special forms of a parallelogram, and square as a special form of a rhombus and a rectangle. In other words, PMSMT 20 was able to classify all quadrilaterals correctly.

\section{Dual Inclusion Relations of Quadrilaterals}

When PMSMTs' answers on the true-false questions on the pre-test were examined, it was seen that PMSMTs had some problems related to the dual inclusion relations among the quadrilaterals. On the other hand, when PMSMTs work on the post-test was examined, it was seen that almost all of them correctly identified the questions regarding the dual inclusion relation questions as true or false, and rewrote the correct versions of the false statements on the post-test. That is, they enhanced their knowledge of the dual relations among the quadrilaterals through methods course.

To illustrate, nine of the 20 PMSMTs incorrectly answered the question including inclusion relation between a rhombus and a trapezoid as false on the pre-test. The example answer of PMSMT 5 is provided in Figure 7, in which she wrote "(Rhombus) has two pairs of parallel sides and trapezoid has exactly one pair of parallel sides."

On the other hand, all of the PMSMTs correctly answered true-false question regarding the inclusion relation between a rhombus and a trapezoid as true on the post-test.

Two of the PMSMTs incorrectly answered the true-false question "All trapezoids are parallelograms." as true on the pre-test. On the other hand, all of the PMSMTs correctly answered this question as false and corrected the statement as 'all parallelograms are trapezoids' on the post-test. 


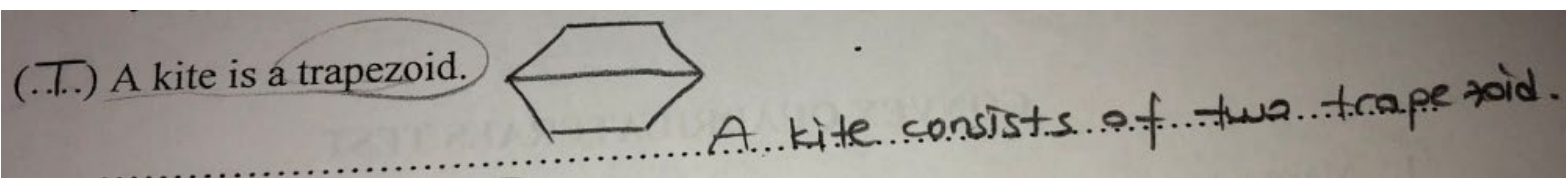

Figure 8. PMSMT 15's answer related to kite-trapezoid relationship on the pre-test

(F) A kite is a trapezoid.

Kite is a convex quadrilateral.

Figure 9. PMSMT 15's answer related to kite-trapezoid relationship on the post-test

Six of the PMSMTs incorrectly answered the true-false question "A kite is a trapezoid." as true on the pre-test. The sample answer of PMSMT 15 is provided in Figure 8.

As can be understood from Figure 8, PMSMT 15 confused a kite with a hexagon andincorrectly stated that "a kite consists of two trapezoids." Due to this confusion, she incorrectly identified the statement "a kite is a trapezoid" as true.

On the other hand, 17 (out of 20) of the PMSMTs correctly answered the true-false question "a kite is a trapezoid" as false on the post-test. The sample answer of PMSMT 15, for which she rewrote the statement as "Kite is a convex quadrilateral.", is provided in Figure 9.

\section{Definitions of Quadrilaterals}

Regarding the third theme, PMSMTs' answers related to identification, definition, and drawing an alternative shape to the given shape on the pre-test were also analyzed. This analysis indicated that most of the PMSMTs defined each shape by writing most of the properties of the given shapes when they were asked to provide minimal definitions for the quadrilaterals. In other words, they described the properties of the given shapes without paying attention to whether or not there were extra information that would be obtained from other properties. In addition, the findings also showed that, while describing the quadrilaterals, they did not focus on the inclusion relations among the quadrilaterals. Besides, it was revealed that almost all of the PMSMTs drew alternative shapes to the given shapes by translating those shapes or drew the prototypes of the given shapes. On the other hand, when PMSMTs' answers on the post-test were examined, it was seen that 16 of the PMSMTs were able to identify the quadrilaterals (even if they were represented other than their prototypical figures) as correct and drew possible alternative shapes other than prototypical figures. These 16 PMSMTs were also able to define each shape by using inclusion relations and referring to the necessary and sufficient (De Villiers, 1994) properties correctly on the post test. That is, they enhanced their knowledge regarding identifying, defining, and drawing alternative shapes through the methods course. PMSMT 1's answer on the pre-test is provided in Figure $\mathbf{1 0}$ as an example. 
a) Identify each shape as quadrilaterals. (You can use more than one name for each shape.)

b) Draw an alternative shape to given shape according to your identification.

c) Define each shape according to your identification.

- Shape 1:

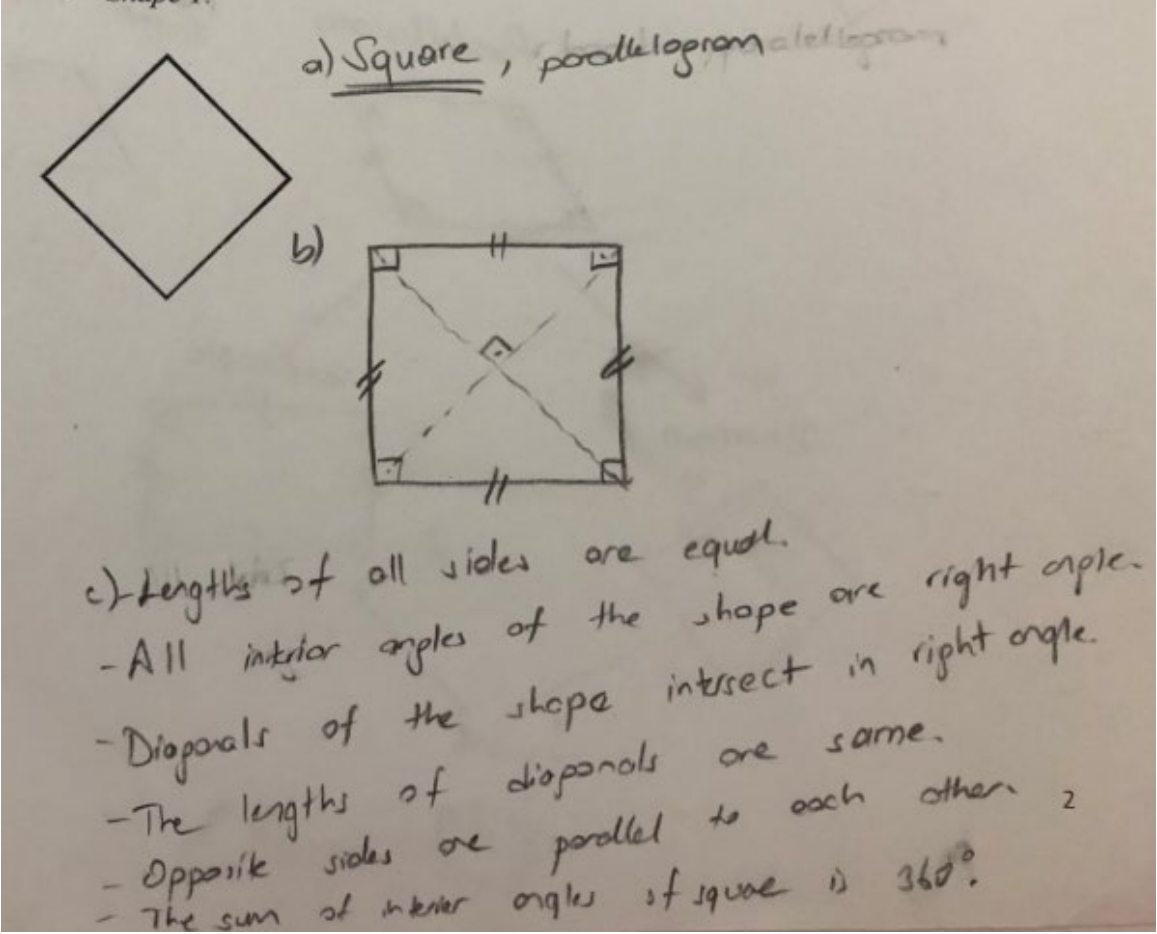

Figure 10. PMSMT 1's answer related to identifying, defining and drawing quadrilaterals on the pretest

As deduced from Figure 10, PMSMT 1 only described the properties of the given figure rather than providing a definition for the given shape and identified it as a square and a parallelogram. Nevertheless, there was no indication of the inclusion relations between a parallelogram and a square and other quadrilaterals in her answer. Also, as an alternative to the given shape, she drew the prototypical figure of a square. PMSMT 1's answer on the post-test is also provided in Figure $\mathbf{1 1}$ as an example.

As deduced from Figure 11, PMSMT 1 was able to identify the given shape by all the possible quadrilaterals (square, rhombus, rectangle, and parallelogram) except a trapezoid, and define it minimally by using inclusion relations between a parallelogram and a square on the post-test. However, she drew prototype of a square as an alternative to the given shape, similar to the pre-test. Another example answer of PMSMT 8 on the pre-test is provided in Figure 12. 
a) Identify each shape as quadrilaterals. (You can use more than one name for each shape.)

b) Draw an altemative shape to given shape according to your identification.

c) Define each shape according to your identification. (Please use minimal definition.)

- Shape 1:

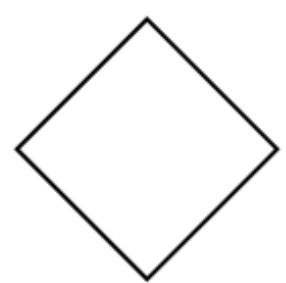

a) It looks like a square, if it is square, it must be rhombus, rectangle, parallelogram, and trapezoid.

b)

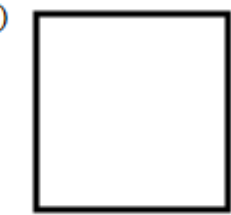

c) Parallelogram with a right angle and all sides are congruent.

Figure 11. The answer of PMSMT 1 on identifying, defining, and drawing quadrilaterals on the posttest

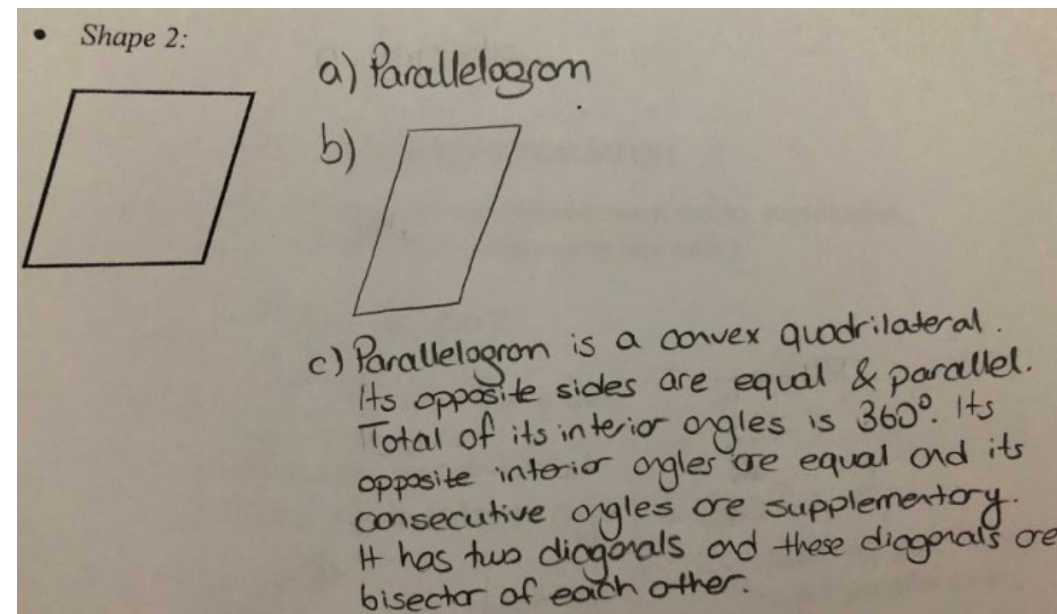

Figure 12. PMSMT 8's answer related to identifying, defining, and drawing quadrilaterals on the pretest

,As understood from Figure 12, PMSMT 8 was able wrote many properties of the given shape instead of defining it minimally and but identify it as a parallelogram. Also, as an alternative to the given shape, she drew a prototypical figure of a parallelogram. PMSMT 8's answer on the post-test is also provided in Figure 13 to be compared with her performance on the pre-test.

As deduced from Figure 13, PMSMT 8 was able to identify the given shape as a parallelogram and define it minimally by using necessary and sufficient characteristics and inclusion relations between a parallelogram and a trapezoid. Also, she drew a rectangle instead of drawing the prototype of a parallelogram. 
- Shape 2:

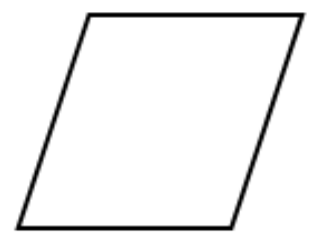

a) Parallelogram

b)

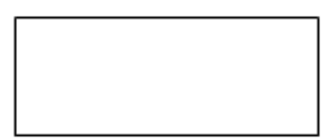

c) A parallelogram is a trapezoid with two pairs of parallel sides.

Figure 13. PMSMT 8's answer related to identifying, defining, and drawing quadrilaterals on the posttest

\section{DISCUSSION AND CONCLUSION}

The purpose of this study was to investigate PMSMTs' (mis)conceptions related to definitions, classifications, and inclusion relations of convex quadrilaterals before and after taking a methods course, focusing on the teaching of geometry conceptually. On the basis of the pre-test results of the study, it was concluded that PMSMTs had several misconceptions related to the classifications and inclusion relations of convex quadrilaterals when they started taking a methods of teaching mathematics course. For instance, based on the analyses of the classifications of quadrilaterals, they did not associate a trapezoid and a kite with other quadrilaterals similar to the findings of Zilkova's (2015) study. In addition, they were not able to classify all the quadrilaterals correctly in a hierarchical way as reported by Erdoğan and Dur (2014). Besides, based on the definitions of quadrilaterals, they were accustomed to the prototypical figures of the quadrilaterals and had problems about definitions in a similar vein with the findings obtained by Fujita and Jones (2006). It was seen that most of the PSMSMTs provided several properties of the quadrilaterals instead of providing the sufficient and necessary properties in order to obtain minimal definitions. Moreover, the findings revealed that they did not make use of the inclusion relations among the quadrilaterals while providing definitions for the quadrilaterals. Moreover, although, prior to the study, the participants of the study had some correct conceptions in dual relations between some quadrilaterals such as square-rectangle and trapezoidparallelogram similar to findings obtained by Zeybek (2017), they had several misconceptions regarding the dual relations between other quadrilaterals (e.g., rhombus-trapezoid, kite-trapezoid, and trapezoidparallelogram).

As contrary to the pre-test results, the post-test results indicated that PMSMTs enhanced their content knowledge in quadrilaterals, their classifications and inclusion relations during a methods of teaching mathematics course as reported by Ulusoy (2016) and Zeybek (2017). To illustrate, most of the PMSMTs drew correct concept maps of quadrilaterals by using hierarchical classifications, constructed minimal definitions correctly rather than listing several properties, and drew alternative shapes not limited to the prototypical shapes to the given quadrilaterals. Moreover, most of the PMSMTs corrected their incorrect answers related to the dual inclusion relations between quadrilaterals on the post-test. When pre- and post-test results were compared, it was concluded that the methods course had a positive impact on developing PMSMTs' content knowledge on classifications, definitions, and inclusion relations of quadrilaterals. This might be resulting from the fact that during the course, pre-service teachers examined and discussed two dimensional and three-dimensional shapes, their properties and definitions by taking inclusion relations into consideration and were engaged in some activities related to the shapes and their properties. More specifically, the inquiry-based approach in the methods course might have helped PMSMTs to organize and improve their knowledge of defining and classifying 
quadrilaterals. This is consistent with the results of other studies, which showed that mathematics methods courses increase pre-service teachers' content knowledge (Burton, Daane, \& Giesen, 2008; Matthews \& Seaman, 2007; Matthews, Rech, \& Grandgenett, 2010; Quinn, 1997). Thus, during method course it is important to create a learning environment in which the instructors support PSMSMTs' learning with question-driven instruction, classroom discussion, and cooperation (Chapman, 2011; Jarrett, 1997).

According to the results of this study, both in the pre- and post-test implementations, PMSMTs were most successful in the inclusion relationship between a square and a rectangle and less successful in classifying a kite and a trapezoid. In particular, it should be noted that although the methods course had a positive impact on supporting the PSMSMTs' content knowledge of quadrilaterals and their hierarchical classifications and definitions, the findings of this study also showed that some of the participants still had misconceptions about classifying shapes and inclusion relations between shapes (e.g., kite and other quadrilaterals) after taking the methods course. Therefore, it is concluded that kite remains to be the most problematic quadrilateral of all. In the Turkish Middle School Mathematics Curriculum (2018), the properties of quadrilaterals appear as a main objective and the dual relationships among the quadrilaterals as a sub-objective. This might result in putting a much more emphasis on the properties of quadrilaterals than their dual inclusion relations. Therefore, it is suggested that a main objective related to the inclusion relations of all quadrilaterals (especially a kite and its relationships with other quadrilaterals) is added to the middle school mathematics curriculum. Furthermore, it is suggested that those relations are discussed, and preservice mathematics teachers are engaged in productive discussions related to the inclusion relations and hierarchical definitions of quadrilaterals during the methods of teaching mathematics courses.

Although this study has the potential to contribute to the body of literature related to PSMSMTs' development of inclusion relations and hierarchical classifications of quadrilaterals through a methods course, this study also has several limitations. First of all, since the participants of the study included only 20 PMSMTs, the results of the study may not be generalized to a larger population. However, it should be noted that generalizing is not the main purpose in case studies. This study focuses on a specific case that included a specific group of participants and a specific concept (i.e., definitions, classifications, and inclusion relations of quadrilaterals) before and after taking a methods course. Nevertheless, the results of the study may be useful for other researchers' and/or mathematics teacher educators if they study with a group of PMSMTs whose traits are similar to the participants in this study. Secondly, this study included only the PMSMTs' written answers to the data collection tool that included true-false and open-ended questions. Therefore, it was not possible to understand the underlying reasons behind PMSMTs' misconceptions and how and in what ways the PMSMTs benefited from taking a methods course. Therefore, it is suggested that interviews be conducted with PMSMTs in order delve into their reasoning about quadrilaterals and their inclusion relations as a scope of a further study. Moreover, studies conducted in other cultural contexts with different teacher education programs with different samples of PSMSMTs might be helpful to compare and contrast participants' content knowledge and its development through a methods course.

Author contributions: All authors were involved in concept, design, collection of data, interpretation, writing, and critically revising the article. All authors approve final version of the article.

Funding: The authors received no financial support for the research and/or authorship of this article.

Declaration of interest: Authors declare no competing interest.

Data availability: Data generated or analysed during this study are available from the authors on request.

\section{REFERENCES}

Akkaş, E. N., \& Türnüklü, E. (2015). Middle school mathematics teachers' pedagogical content knowledge regarding student knowledge about quadrilaterals. Elementary Education Online, 14(2), 744-756. https://doi.org/10.17051/io.2015.12002

Aktaş, D. Y., \& Cansız-Aktaş, M. (2012). Eighth grade students' understanding and hierarchical classification of quadrilaterals. Elementary Education Online, 11(3), 714-728. 
Artigue, M., \& Blomhøj, M. (2013). Conceptualizing inquiry-based education in mathematics. ZDM, 45(6), 797-810. https://doi.org/10.1007/s11858-013-0506-6

Ball, D. L., Thames, M. H., \& Phelps, G. (2008). Content knowledge for teaching. Journal of Teacher Education, 59, 389-407. https://doi.org/10.1177/0022487108324554

Brunheira, L., \& da Ponte, J. P. (2019). From the classification of quadrilaterals to the classification of prisms: An experiment with prospective teachers. The Journal of Mathematical Behavior, 53, 65-80. https://doi.org/10.1016/j.jmathb.2018.06.004

Burton, M., Daane, C. J., \& Giesen, J. (2008). Infusing Mathematics content into a methods course: impacting content knowledge for teaching. https://files.eric.ed.gov/fulltext/EJ835496.pdf

Chapman, O. (2011). Elementary school teachers' growth in inquiry-based teaching of mathematics. ZDM, 43, 951-963. https://doi.org/10.1007/s11858-011-0360-3

Clements, D. H. \& Battista, M. T. (1992). Geometry and spatial reasoning. In D. Grows (Ed.), Handbook of research on mathematics teaching and learning (pp. 420- 464). MacMillan.

De Villiers, M. (1994). The role and function of a hierarchical classification of quadrilaterals. For the Learning of Mathematics, 14(1), 11-18.

Erdoğan, E. O. \& Dur, Z. (2014). Preservice mathematics teachers' personal figural concepts and classifications of quadrilaterals. Australian Journal of Teacher Education, 39(6), 107-133. https://doi.org/10.14221/ajte.2014v39n6.1

Fraenkel, J. R., Wallen, N. E. \& Hyun, H. H. (2012). How to design and evaluate research in education (8th ed.). McGraw-Hill.

Fujita, T. \& Jones, K. (2006). Primary trainee teachers' understanding of basic geometrical figures in Scotland. Proceedings of the 30th Conference of the International Group for the Psychology of Mathematics Education (PME 30), 3, 129-136.

Fujita, T. (2012). Learners' level of understanding of the inclusion relations of quadrilaterals and prototype phenomenon. The Journal of Mathematical Behavior, 31(1), 60-72. https://doi.org/10.1016/j.jmathb.2011.08.003

Fujita, T., \& Jones, K. (2007), Learners' understanding of the definitions and hierarchical classification of quadrilaterals: towards a theoretical framing. Research in Mathematics Education, 9(1\&2), 3-20. https://doi.org/10.1080/14794800008520167

Jarrett, D. (1997). Inquiry strategies for science and mathematics learning. https://files.eric.ed.gov/fulltext/ED413188.pdf

Jaworski, B. (2004). Grappling with complexity: Co-learning in inquiry communities in mathematics teaching development. Proceedings of the 28th conference of the international group for the psychology of mathematics education, Bergen University College, 17-36.

Jones, K., \& Tzekaki, M. (2016). Research on the teaching and learning of geometry. In A. Gutiérrez, G. Leder, \& P. Boero (Eds.), The second handbook of research on the psychology of mathematics education (pp. 109-149). Sense Publishers. https://doi.org/10.1007/978-94-6300-561-6_4

Karakuş, F., \& Erşen, Z. B. (2016). Examining pre-service primary school teachers' definitions and classifications toward quadrilaterals. Karaelmas Journal of Educational Sciences, 4, 38-49.

Loc, N. P., Tong, D. H., \& Hai, N. T. B. (2017). The investigation of primary school students' ability to identify quadrilaterals: a case of rectangle and square. The International Journal of Engineering and Science (IJES), 6(3), 93-99. https://doi.org/10.9790/1813-0603019399

Matthews, M. E., \& Seaman, W. I. (2007). The effects of different undergraduate mathematics courses on the content knowledge and attitude towards mathematics of preservice elementary teachers. https://files.eric.ed.gov/fulltext/EJ835494.pdf

Matthews, M., Rech, J., \& Grandgenett, N. (2010). The impact of content courses on pre-service elementary teachers' mathematical content knowledge. Teacher Education Faculty Publications. https://digitalcommons.unomaha.edu/tedfacpub/22

Ministry of National Education [MoNE]. (2018). Matematik dersi öğretim programı (ilkokul ve ortaokul 1,2,3,4,5, 6, 7 ve 8. sinıflar) [Mathematics curriculum (for grades 1,2,3,4,5, 6, 7, and 8)]. MEB.

National Council of Teachers of Mathematics (NCTM). (2000). Principles and standards for school mathematics.

Özkan, M., \& Bal, A. P. (2017). Analysis of the misconceptions of 7th grade students on polygons and specific quadrilaterals. Eurasian Journal of Educational Research, 6, 161-182. https://doi.org/10.14689/ejer.2017.67.10

Öztoprakçı, S. (2014). Pre-service middle school mathematics teachers' understanding of quadrilaterals through the definitions and their relationships (Unpublished Doctoral Dissertation). Middle East Technical University, Ankara, Turkey.

Park, S., \& Oliver, J. S. (2008). Revisiting the conceptualization of pedagogical content knowledge (PCK): PCK as a conceptual tool to understand teachers as professionals. Research in Science Education, 38, 261-284. https://doi.org/10.1007/s11165-007-9049-

Quinn, R. J. (1997). Effects of mathematics methods courses on the mathematical attitudes and content knowledge of preservice teachers. The Journal of Educational Research, 91(2), 108-114. https://doi.org/10.1080/00220679709597528

Shulman, L. S. (1986). Those who understand: knowledge growth in teaching. Educational Researcher, 15(2), 4-14. https://doi.org/10.3102/0013189X015002004

Shulman, L. S. (1987). Knowledge and teaching: foundations of the new reform. Harvard Educational Review, 5(1), 1-22. https://doi.org/10.17763/haer.57.1.j463w79r56455411 
Smith, M. E., Swars, S. L., Smith, S. Z., Hart, L. C. \& Haardörfer, R. (2012) Effects of an additional mathematics content course on elementary teachers' mathematical beliefs and knowledge for teaching. Action in Teacher Education, 34(4), 336-348. https://doi.org/10.1080/01626620.2012.712745

Türnüklü, E. (2014). Construction of inclusion relations of quadrilaterals: analysis of pre-service elementary mathematics teachers' lesson plans. Education and Science, 39(173), 198-208.

Ulusoy, F. (2016). Developing prospective mathematics teachers' knowledge for teaching quadrilaterals through a video case-based learning environment (Unpublished Doctoral Dissertation). Middle East Technical University, Ankara, Turkey.

van de Walle, J. A., Karp, K. S., \& Bay-Williams J. M. (2013). Elementary and middle school mathematics: Teaching developmentally (8th Ed.). Pearson Education, Inc.

Yin, R. K. (2003). Case study research design and methods. Sage.

Youngs, P., \& Qian, H. (2013). The influence of university courses and field experiences on Chinese elementary candidates' mathematical knowledge for teaching. Journal of Teacher Education, 64(3), $244-261$. https://doi.org/10.1177/0022487112473836

Zeybek, Z. (2017). Learning to understand inclusion relations of quadrilaterals. The Eurasia Proceedings of Educational \& Social Sciences (EPESS), 6, 9-13.

Zilkova, K. (2015). Misconceptions in pre-service primary education teachers about quadrilaterals. Journal of Education, Psychology and Social Sciences, 3(1), 1339-1488. 


\section{APPENDIX}

\section{Convex Quadrilaterals Test}

1. Make a concept map in order to show relationships between convex quadrilaterals.

2. Identifying each statement as True or False. Correct wrong statements and rewrite.

(....) A kite is a trapezoid.

(....) Every rectangle is a square.

(....) All squares are parallelograms.

(....) All trapezoids are parallelograms.

(....) If it is a rhombus, then it is a trapezoid.

(....) If it has exactly two lines of symmetry, it must be a quadrilateral.

3. a) Identify each shape as quadrilaterals. (You can use more than one name for each shape.)

b) Draw an alternative shape to given shape according to your identification.

c) Define each shape according to your identification. (Please use minimal definition.)

- Shape 1:

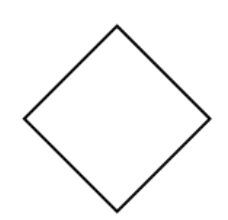

- Shape 2:

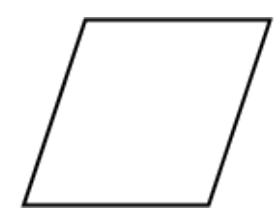

- Shape 3:

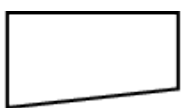

4. Draw a trapezoid. 